\title{
Maturation des spermatozoïdes de bélier. Etude préliminaire du mouvement flagellaire caractéristique des formes de transition du corps de l'épididyme
}

\author{
C. CHEVRIER, J. L. DACHEUX
}

Laboratoire de Physiologie Comparée, Faculté des Sciences, 37200 Tours, France.

Laboratoire de Physiologie de la Reproduction, I.N.R.A., 37830 Monnaie.

Summary. Maturation of ram spermatozoa. Preliminary study of the flagellar movement characteristic of the transitory forms of the epididymal corpus.

Mammalian spermatozoa acquire the ability to move forward during epididymal maturation and the corpus occurs as a critical region where all the patterns of movements can be observed: immature, 'erratic' and mature. The absence of coordination of the flagellar movement of the transitory forms provides a difference in the initiation sites and the waves propagation between the two sides of the axonema. The mechanisms that may be required to activate selectively the tubulin-dynein system on the two sides of the flagellum during the initiation of the movement of immature sperm in mammals are unknown.

\section{Introduction.}

Contrairement à la plupart de ceux des Invertébrés, les spermatozoïdes de Mammifères à la sortie du testicule ne sont pas fécondants. Ils n'acquièrent leur capacité à féconder qu'au cours du transit dans l'épididyme. L'acquisition de l'aptitude à se déplacer est un facteur déterminant de la maturation des spermatozoïdes; elle s'effectue graduellement au cours du transit.

Dans cette étude, nous avons déterminé chez le Bélier les différentes formes de motilité qui caractérisent la mise en place du mouvement progressif des spermatozoïdes au cours de leur transit épididymaire. Une analyse du mouvement flagellaire correspondant à des motilités transitoires a été effectuée.

\section{Matériel et méthodes.}

Les expériences ont été réalisées sur des Béliers Ile-de-France d'âge adulte. Les spermatozoïdes épididymaires ont été obtenus par microperfusion de 10 régions de l'organe après abattage de l'animal, selon la technique de Dacheux 
et al. (1983). Les échantillons ont été dilués à une concentration finale de 10-50 $10^{6}$ spermatozö̈des $/ \mathrm{ml}$ dans du tampon phosphate salin (PBS). La motilité des spermatozoïdes a été étudiée après $10 \mathrm{~min}$ d'incubation à $37^{\circ} \mathrm{C}$ par observation microscopique d'une suspension cellulaire placée entre lame et lamelle. La détermination des différents modèles de motilité et l'analyse du mouvement flagellaire ont été effectuées sur clichés photographiques obtenus par la technique de «Multiple Moving Exposure» (Chevrier et Dacheux, 1987).

\section{Résultats.}

Au cours du transit épididymaire le mouvement des spermatozoïdes évolue (tabl. 1). Dans la région antérieure de l'épididyme, le mouvement flagellaire est seulement représenté par des oscillations sans propagation d'ondes (mouvement de type A) ; les spermatozoïdes apparaissent fortement incurvés avec partiellement un léger mouvement vibratoire. En aucun cas les spermatozoïdes prélevés dans cette région ne se déplacent. Dans le corps de l'épididyme $30 \%$ des spermatozoïdes présentent un mouvement flagellaire de ce type, $50 \%$ développent des mouvements circulaires et erratiques caractéristiques de formes transitoires, tandis que $20 \%$ d'entre eux ont un mouvement flagellaire coordonné permettant une progression rectiligne analogue à celle de spermatozoïdes matures (Chevrier et Dacheux, 1987).

\section{TABLEAU 1}

Evolution des formes de mouvements des spermatozoïdes au cours du transit épididymaire.

Régions

épididymaires

5
6
7
8
$9-10$

$\%$ des différents modèles de motilité

\begin{tabular}{rrrr}
\hline A & B & C & D \\
\hline 67 & 21 & 12 & 0 \\
29 & 29 & 22 & 20 \\
11 & 5 & 4 & 80 \\
16 & 4 & 5 & 75 \\
8 & 1 & 2 & 89
\end{tabular}

A : Oscillations flagellaires seulement; $B$ et $C$ : Mouvements flagellaires non coordonnés ; D : Mouvements flagellaires synchronisés de spermatozoïdes progressifs.

Le mouvement des spermatozoïdes prélevés dans le corps de l'épididyme et présentant un mouvement de type transitoire (fig. 1) est caractérisé par l'existence de grandes courbures (angle égal ou supérieur à 3 radians) principalement

FIG. 1. - Photomicrographie d'un spermatozoide du corps de l'épididyme de Bélier avec un mouvement de type transitoire obtenu par la technique MME (multiple moving exposure) (Chevrier et Dacheux, 1987). Le temps d'exposition est de 1/8 sec sous illumination stroboscopique (100 flashes $\cdot \mathrm{sec}^{-1}$ ). La barre d'échelle représente $10 \mu \mathrm{m}$. 


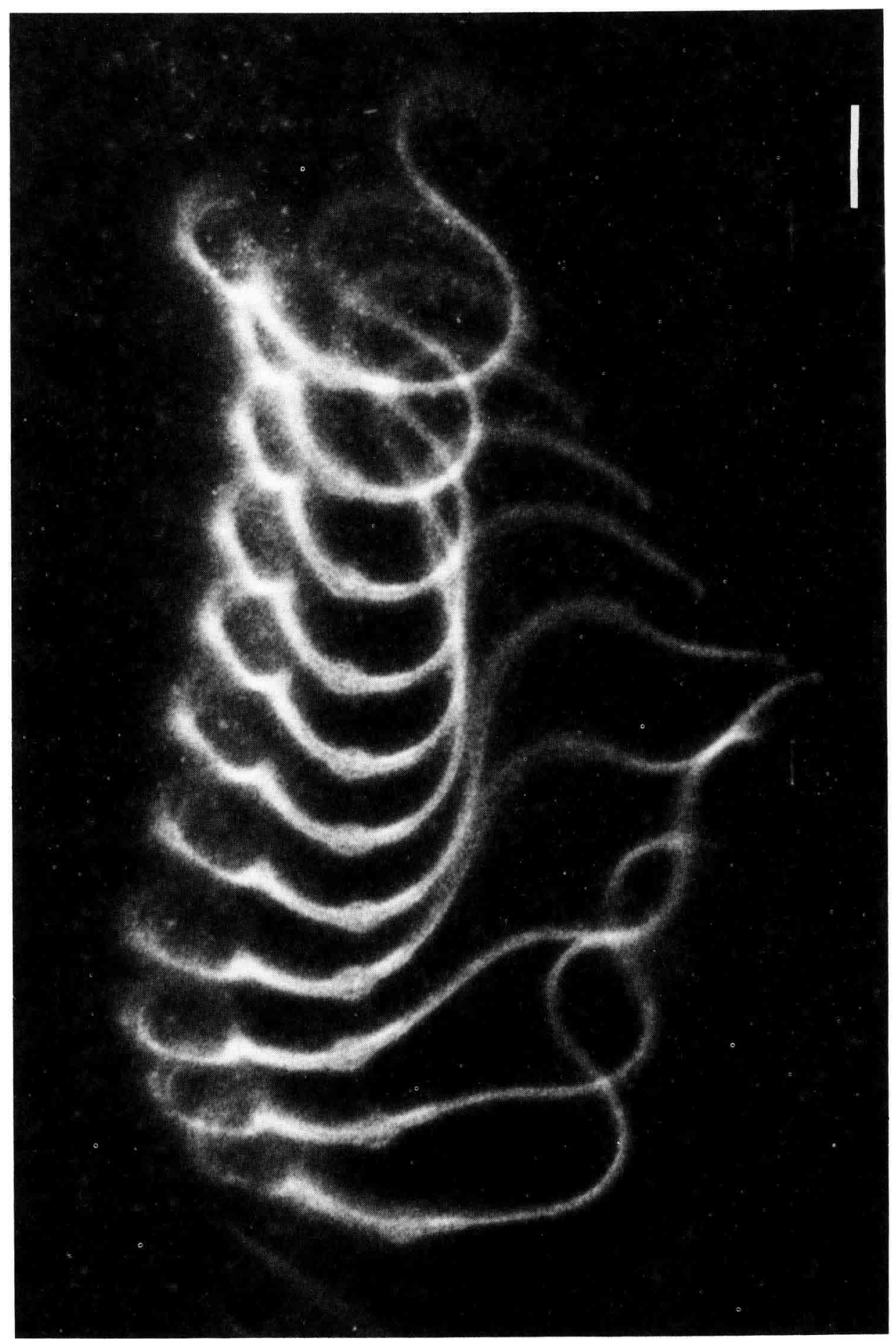


localisées dans la première partie du flagelle. En utilisant pour représenter ces angulations la méthode de construction des courbes de contraintes décrite par Brokaw et al. (1982) et Gibbons (1981) (angulation du flagelle calculée par rapport à l'axe de symétrie du noyau), on constate la présence à la fin de la pièce intermédiaire d'une courbure non propagée et l'existence d'ondes successives (principale et reverse) sur le reste du flagelle. Ceci se traduit sur les courbes de contraintes (fig. 2) par la présence d'une angulation constante d'environ 0,30 radians à la base du flagelle. L'onde principale naît à la base du flagelle alors que l'onde reverse prend naissance au niveau de la pièce intermédiaire quand la courbure principale du flagelle a atteint sa valeur maximale (environ 3,8 rad). L'onde principale demeure à la base du flagelle pendant la plus grande période de battement et atteint un développement maximal de $0,1 \mathrm{rad} \cdot \mu \mathrm{m}^{-1}$. Elle se propage quand l'onde reverse est initialisée.

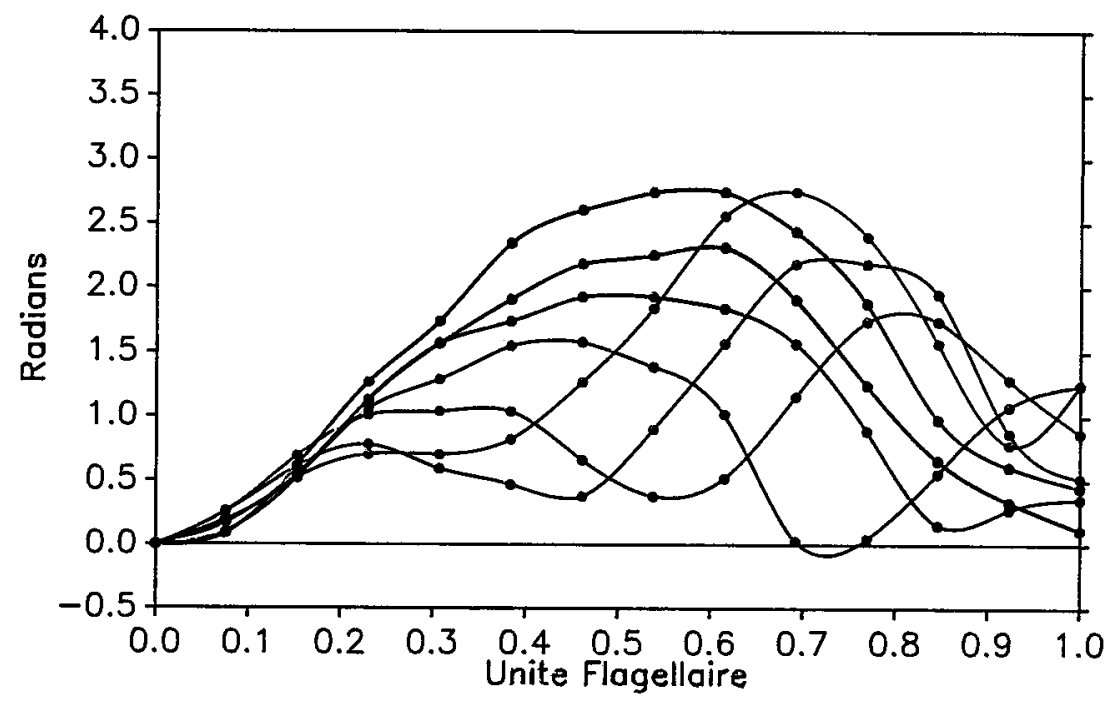

FIG. 2. - Courbes de contraintes du spermatozoïde présenté en figure 1. Les méthodes de construction de ces courbes ont été développées par Brokaw et al. (1982) et Gibbons (1981). L'onde principale est définie comme une région de pente positive et l'onde reverse comme une région de pente négative. L'intervalle entre deux courbes successives est de $10 \mathrm{msec}$. La longueur du flagelle est de $59,5 \mu \mathrm{m}$.

Les différences observées entre les taux de glissement (estimés par l'écart entre deux courbes de contraintes successives (Brokaw et al., 1982)) dans la direction de l'onde principale et la direction de l'onde reverse traduisent des différences dans les vitesses de propagation des ondes selon les 2 moitiés de l'axonème.

\section{Conclusion.}

Le corps de l'épididyme est une région critique dans la mise en place du mouvement flagellaire. Dans cette région, toutes les formes de mouvements sont 
observées. Cependant la majeure partie de la population est représentée par des mouvements flagellaires de type transitoire caractérisés par des déplacements circulaires et erratiques. Pour ces spermatozoïdes, le manque de coordination du mouvement aboutit à la présence de courbures asymétriques du flagelle. Des différences de localisation du site de naissance des ondes et de leur vitesse de propagation selon les deux côtés de l'axonème conduisent à donner aux spermatozoïdes un mouvement désordonné caractéristique. Le mécanisme mis en jeu pour coordonner symétriquement les courbures flagellaires selon les deux côtés de l'axonème est inconnu.

$5^{e}$ Congrès de la Société d'Andrologie de langue française, Paris, décembre 1987.

Remerciements. - Travaux subsidiés par le contrat INSERM, $n^{\circ} 854006$.

\section{Références}

BROKAW C. J., LUCK D. J. L., HUANG B., 1982. Analysis of the movement of chlamydomonas flagella: the function of the radial spoke system is revealed by comparison of wild-type and mutant flagella. J. Cel/ Biol., 92, 722-732.

CHEVRIER C., DACHEUX J. L., 1987. Analysis of the flagellar bending waves of ejaculated ram sperm. Cell Mot. Cytoskel., 8, 261-273.

DACHEUX J. L., PAQUIGNON M., COMBARNOUS Y., 1983. Head-to-head agglutination of ram and boar epididymal spermatozoa and evidence for an epididymal antagglutinin. J. Reprod. Fert., 67, $181-189$.

GIBBONS I. R., 1981. Transient flagellar waveforms during intermittent swimming in sea urchin sperm. II. Analysis of tubule sliding. J. Musc. Res. Cell Motil. 2, 83-130. 